\title{
Integrated Filtering Microstrip Duplex Antenna Array with High Isolation
}

\author{
Xian-Jing Lin, Ze-Ming Xie, and Pei-Sheng Zhang \\ School of Electronic and Information Engineering, South China University of Technology, Guangzhou, China \\ Correspondence should be addressed to Xian-Jing Lin; eexjlin@mail.scut.edu.cn
}

Received 27 November 2016; Accepted 23 January 2017; Published 22 February 2017

Academic Editor: Jaume Anguera

Copyright ( 2017 Xian-Jing Lin et al. This is an open access article distributed under the Creative Commons Attribution License, which permits unrestricted use, distribution, and reproduction in any medium, provided the original work is properly cited.

\begin{abstract}
This paper presents a $2 \times 1$ integrated filtering microstrip duplex antenna array with high isolation and same polarization. The antenna consists of two radiating patches fed by two T-shaped probes and a power distributing duplex network (PDDN). The PDDN is composed of two bandstop filters and a 180-degree phase shift power divider. And the PDDN is designed to achieve the functions of power division, frequency selectivity, and port isolation. A Transmission Line (TL) model is adopted to design the PDDN, and the detailed synthesis procedure is presented. For demonstration, the proposed antenna is designed and fabricated. The implemented antenna achieves an average gain of $10 \mathrm{dBi}$, a cross-polarization ratio of $20 \mathrm{~dB}$, and an isolation of $35 \mathrm{~dB}$ within the operation band.
\end{abstract}

\section{Introduction}

Full-duplex communication is one of the most important operating modes in modern wireless communications. It is common to use antennas cascaded with duplexes or filters to realize full-duplex communication. However, antennas, duplexes, or filters are designed separately and cascaded through $50 \mathrm{ohm}$ interfaces and matching networks. Due to the different bandwidths of the duplexer, filter, and the antenna, they are usually not well matched; then it will degrade the performances of the system. And it will enlarge the size and weight of the overall system, too.

One of the effective methods to improve the efficiency and reduce the size and weight of the system is to integrate the designs of antennas, duplexes, and filters. Therefore, designs of duplex antennas have attracted much attention of researchers. Using different feeding networks to transmit transmitting (Tx) and receiving ( $\mathrm{Rx}$ ) signals to two stacked patches is a common way to realize duplex function [1]. However, the antenna suffers from narrow bandwidth and complicated configuration. Dual-polarized antenna is another effective method to achieve duplex operation. It transmits and receives signals through a common patch to make the antenna more compact. In [2], a dual-polarized filtering antenna array with enhanced bandwidth and selectivity is proposed by integrating the multimode resonators and radiating patches.

Recently, the design of duplex antenna with single polarization is attracting much attention. In [7], a single polarization T-stubs loaded aperture duplex antenna is designed. In [8], the duplex operation is realized by SIW technique. Two separated feeding lines excite the cavity backed bowtie shaped slot to obtain two impervious modes. In [3], a filtering diplexing antenna is designed. It utilizes coupled microstrip resonators and multimode patch to realize duplex function. In [4], two filtering antenna subarrays are integrated together to work as a duplex antenna. The out-of-band radiations of the subarrays are suppressed by adding shorting pins into the filtering elements. The isolation of the antenna is improved. But the size becomes large. Integrating the duplexer and a dual-band antenna can also realize the duplex operation [5]. Though the abovementioned single polarization duplex antennas are well worked, they sustain the disadvantages of narrow bandwidth, low gain, and dissatisfied isolation.

In this paper, a duplex antenna array with same polarization, high isolation, high gains, and acceptable working bandwidths is designed. Bandstop filters and power 


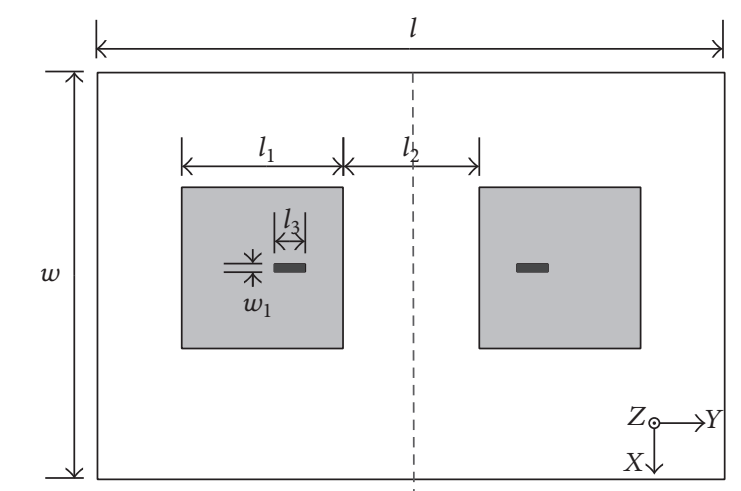

(a)

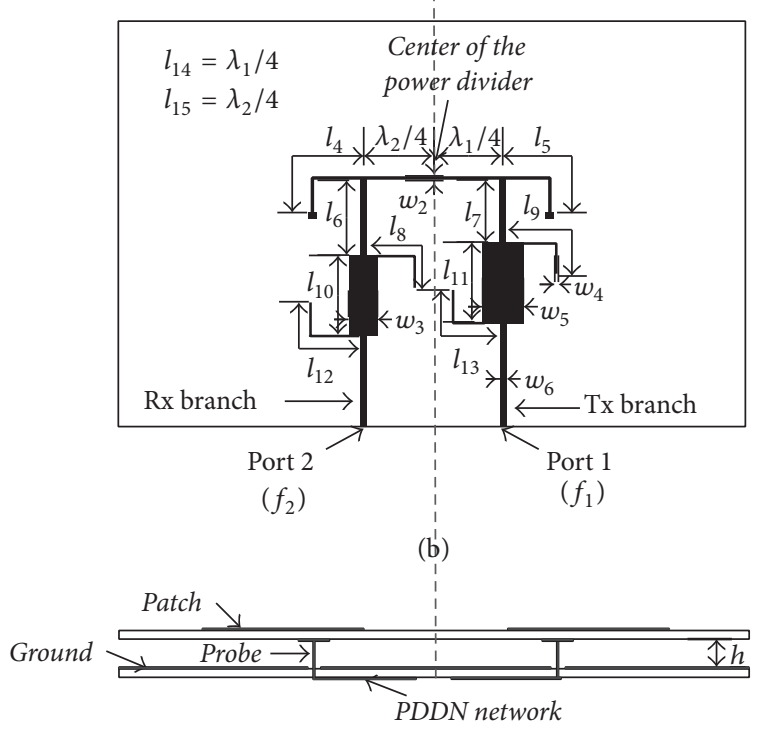

(c)

FIGURE 1: Geometry of the antenna array: (a) top view of the upper substrate, (b) the PDDN, (c) sectional drawing of the antenna array.

divider are adopted to make up a PDDN for a $2 \times 1$ microstrip antenna array. High isolation is obtained by the transmission zeros that produced by the bandstop filters. High gains are realized by the common radiation of the two patch elements. This paper is organized as follows: Section 2 illustrates the geometry and the design of the proposed antenna array. Section 3 presents a comparison between the simulation and measurement results. Section 4 is a conclusion.

\section{Geometry and Design of the Antenna}

2.1. Geometry of the Antenna Array. Figure 1 illustrates the configuration of the proposed antenna array. It consists of two radiating patch elements, a pair of T-shaped probes, and a feeding PDDN. With reference to the figure, the square patch elements with a side length of $l_{1}$ and spacing of $l_{2}$ are fabricated on the top face of the sup-substrate, the ground plane and the feeding circuit are fabricated on the top and bottom faces of the sub-substrate, respectively. Both substrates are with dielectric constant 2.55 , thickness $0.8 \mathrm{~mm}$,
TABLE 1: Dimensions of the proposed antenna (unit: $\mathrm{mm}$ ).

\begin{tabular}{lc}
\hline Symbol & Quantity \\
\hline$l$ & 200 \\
$l_{3}$ & 6.5 \\
$l_{6}$ & 24 \\
$l_{9}$ & 26.5 \\
$l_{12}$ & 26.5 \\
$l_{15}$ & 21.775 \\
$w_{2}$ & 1.27 \\
$w_{5}$ & 13 \\
$l_{1}$ & 49 \\
$l_{4}$ & 28.5 \\
$l_{7}$ & 20 \\
$l_{10}$ & 25.7 \\
$l_{13}$ & 25.9 \\
$w$ & 130 \\
$w_{3}$ & 7 \\
$w_{6}$ & 2.25 \\
$l_{2}$ & 49.5 \\
$l_{5}$ & 27.3 \\
$l_{8}$ & 25.7 \\
$l_{11}$ & 25.5 \\
$l_{14}$ & 22.725 \\
$w_{1}$ & 2 \\
$w_{4}$ & 2.5 \\
$h$ & 6 \\
\hline
\end{tabular}

and loss tangent 0.003 . An air gap with height of $h$ and a pair of probes are introduced between the two substrates. The T-shaped probe consists of a horizontal stripline printed on the back side of the top substrate and a vertical metalized arm. One end of the vertical metalized arm is connected to the center of the horizontal stripline and the other end is connected to the PDDN through a circle slot etched on the ground plane and a via-hole in the sub-substrate. Figure 1(b) shows the PDDN. The PDDN composes of a power divider and two branch circuits connected to the Tx (port 1) and $\mathrm{Rx}$ (port 2) ports. When Tx port is excited, signal of Tx band is split into two equal magnitude and out-of-phase components coupled to the two radiating patches. Exciting the Rx port, same function can be obtained. The out-of-phase operation is realized by the $\lambda / 2$ microstrip lines difference between the distances of the input port and two probes. Note that $\lambda$ is the wavelength of passband center frequency. The Tx and Rx branches are both composed of a bandstop filter and an impedance transformer. The bandstop filter in Tx branch has the passband at the Tx band and the stopband at the Rx band, and vice versa. The overall size of the antenna array is $200 \times 130 \times 7.6 \mathrm{~mm}^{3}$. The detailed dimensions of the proposed antenna array are listed in Table 1.

2.2. Design of the Bandstop Filter (BSF). The BSF is shown in Figure 2(a). It comprises two open-circuited uniform 


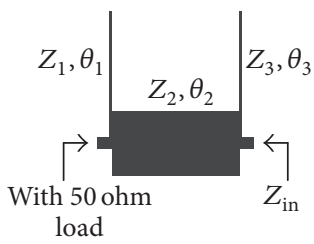

(a)

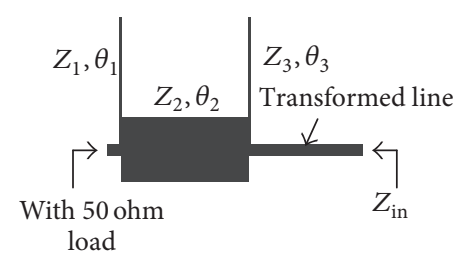

(b)

FIGURE 2: Layout of the BSF: (a) without a transformed line; (b) with a transformed line.

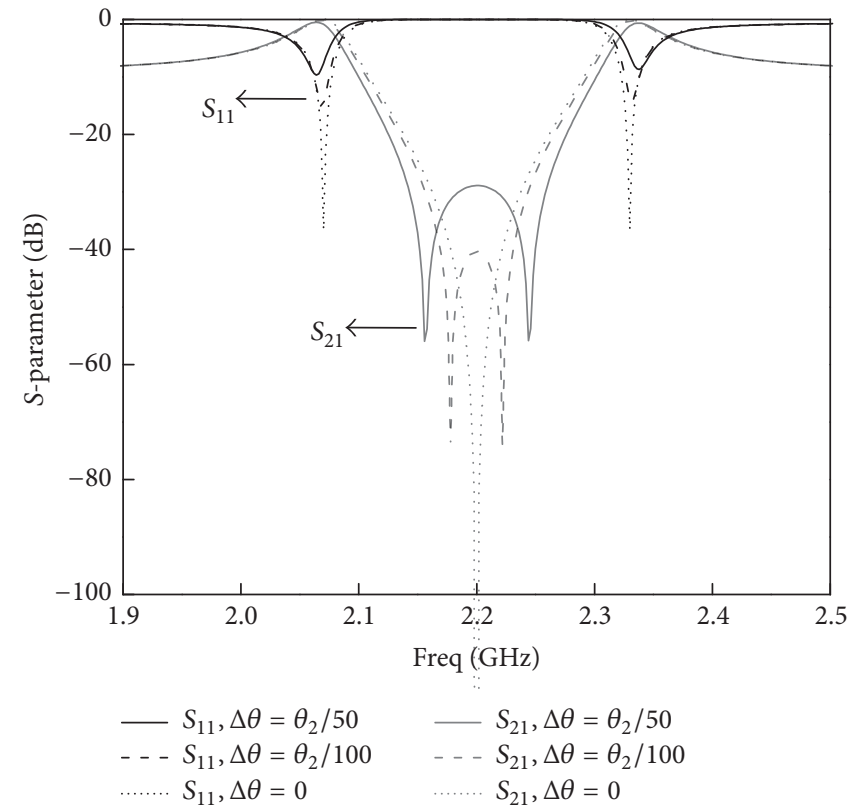

(a)

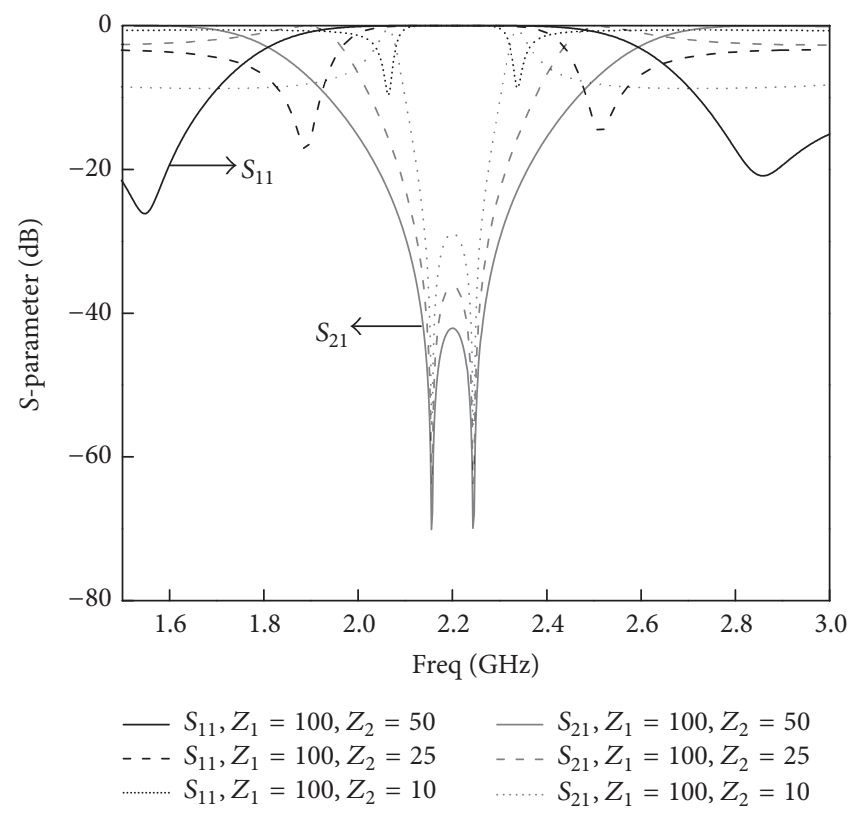

(b)

Figure 3: Effect of the $\Delta \theta$ and impedance ratio. (a) $\Delta \theta$; (b) impedance ratio (in (a), $Z_{1}=100 \Omega, Z_{2}=10 \Omega, \theta_{2}=\pi / 2$; in (b), $\theta_{2}=\pi / 2$, $\left.\Delta \theta=\theta_{2} / 50\right)$.

impedance stubs (UISs) $\left(Z_{1}, \theta_{1}, Z_{3}, \theta_{3}\right)$ and a low impedance $\left(Z_{2}, \theta_{2}\right)$ TL. The $A B C D$ parameters of the BSF are derived using the lossless TL model as

$$
\begin{aligned}
& A=\cos \theta_{2}-K Z_{2} \sin \theta_{2}, \\
& B=j Z_{2} \sin \theta_{2}, \\
& C=j\left(R \cos \theta_{2}+\frac{\sin \theta_{2}}{Z_{2}}-K R Z_{2} \sin \theta_{2}+K \cos \theta_{2}\right), \\
& D=-R Z_{2} \sin \theta_{2}+\cos \theta_{2},
\end{aligned}
$$

where

$$
\begin{gathered}
R=\frac{\tan \theta_{1}}{Z_{1}}, \\
K=\frac{\tan \theta_{3}}{Z_{3}} .
\end{gathered}
$$

Then, the corresponding transmission coefficient and the reflection coefficient of the filter are as follows:

$$
\begin{aligned}
& S_{11}=\frac{A+B / Z_{0}-C Z_{0}-D}{A+B / Z_{0}+C Z_{0}+D}, \\
& S_{21}=\frac{2}{A+B / Z_{0}+C Z_{0}+D} .
\end{aligned}
$$

$Z_{0}$ is the port impedance. According to [6], the transmission zeros are produced by the UISs. To simplify the analysis, we set $\theta_{1}=\theta_{2}-\Delta \theta, \theta_{3}=\theta_{2}+\Delta \theta, Z_{1}=$ $Z_{3}$. Figure 3(a) shows the simulated $S_{11}$ and $S_{21}$ of the BSF with different $\Delta \theta$. Note that it can be seen that $\Delta \theta$ has a significant impact on the transmission zeros of the BSF. Figure 3(b) shows the simulated $S_{11}$ and $S_{21}$ of the BSF with different impedance ratio. The stopband of the BSF will shift to low band when impedance ratio increases. Adjusting the $\Delta \theta$ and impedance ratio, a BSF with proper bandpass and stopband can be obtained. Here, a passband at $2.2 \mathrm{GHz}$ and stopband at $2.4 \mathrm{GHz}$ Tx BSF and a passband at $2.4 \mathrm{GHz}$ and stopband at $2.2 \mathrm{GHz} \mathrm{Rx}$ BSF are designed. To eliminate the mutual interference between the $\mathrm{Tx}$ and $\mathrm{Rx}$ branches, a 


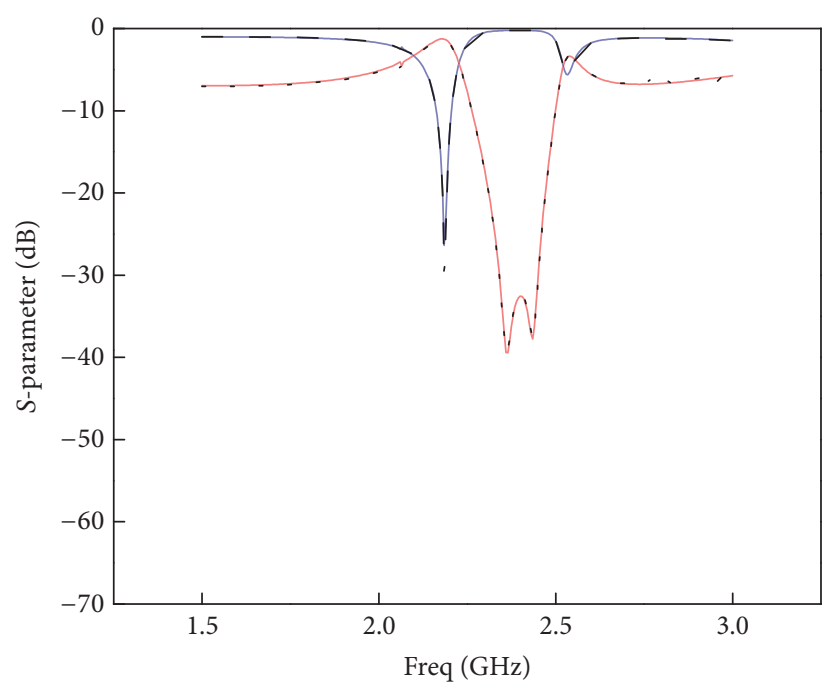

$S_{11}$, without transformer $\quad--S_{11}$, with transformer
$S_{12}$, without transformer $\quad-S_{12}$, with transformer

(a)

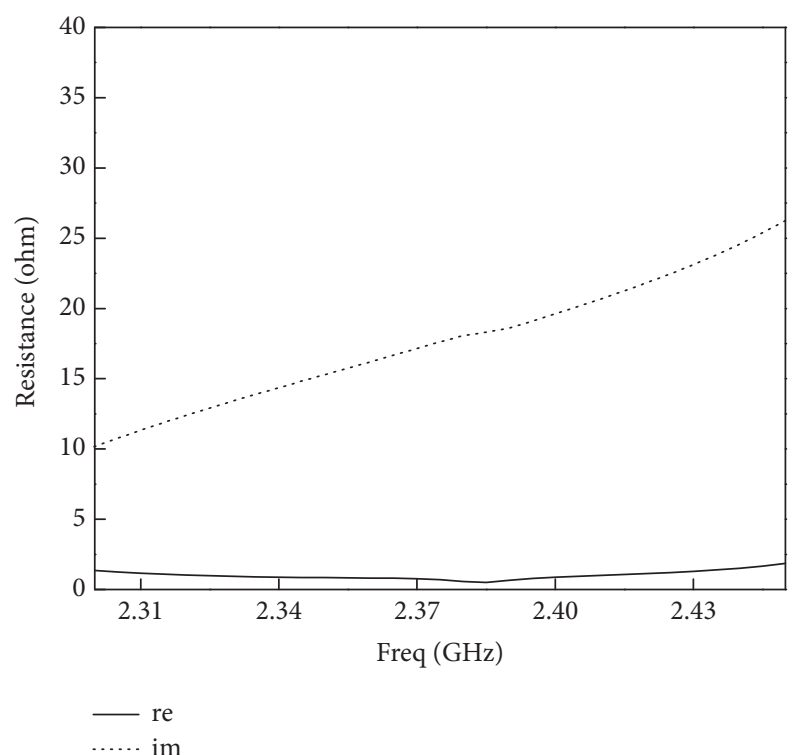

(b)

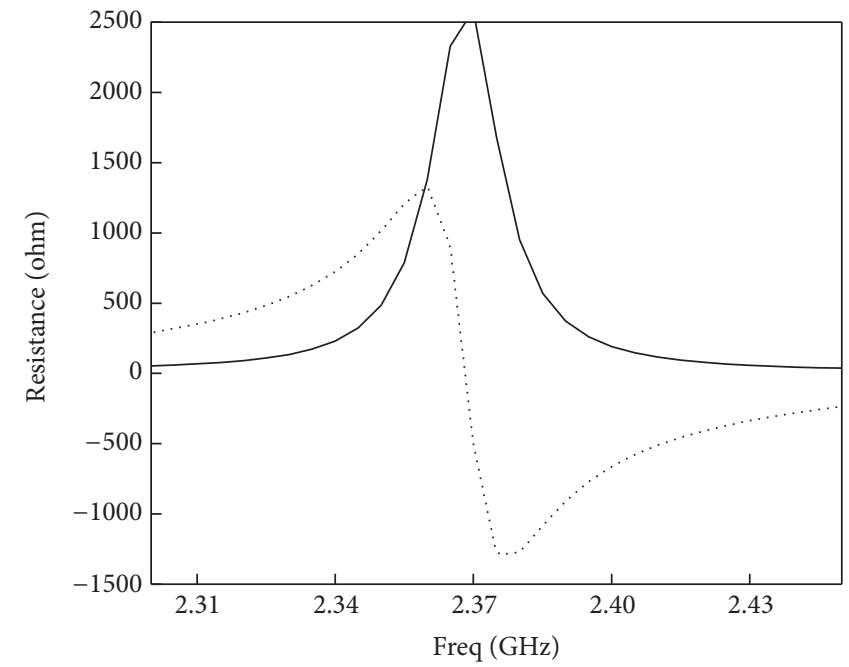

- re

(c)

FIGURE 4: Simulated $S$-parameter and input impedance of Tx BSF: (a) $S$-parameter of the BSFs with and without transformer, (b) input impedance $Z_{\text {in }}$ of the BSF without transformer, (c) input impedance $Z_{\text {in }}$ of the BSF with transformer $\left(Z_{1}=100 \Omega, Z_{2}=10 \Omega, \theta_{2}=\pi / 2\right.$, $\left.\theta_{2}=\pi / 2, \Delta \theta=\theta_{2} / 50\right)$.

$\lambda / 4$ transformed line is employed as shown in Figure 2(b), wherein $\lambda$ is the wavelength of the stopband. The $\mathrm{Tx}(\mathrm{Rx})$ BPS together with the transformed line can be equivalent to open circuit at the $\mathrm{Rx}(\mathrm{Tx})$ band. The $S$-parameter and the input impedance $Z_{\text {in }}$ of the Tx BSF with and without $\lambda / 4$ transformed line are shown in Figure 4. It can be seen that the $S$-parameter is not affected, but the value of $Z_{\text {in }}$ at $2.4 \mathrm{GHz}$ is very large; thus the BSF with $\lambda / 4$ transformed line can be equivalent to terminal open. The input impedance of the $\mathrm{Rx}$ BSF with $\lambda / 4$ transformed line can also be equivalent to open circuit at $2.2 \mathrm{GHz}$. Consequently, the Tx and Rx operation can work independently.
2.3. Design of the PDDN. Figures 5(a) and 5(b) show the configuration of the power divider and the PDDN, respectively. The power divider is a symmetrical structure like a $\mathrm{C}$ shape. When $\Delta l$ is about quarter wavelength of the operating center frequency of the input port, signals from input port (port 1) can split two equal magnitude and out-of-phase components to output ports (port 3 and port 4).

The two equal magnitude and out-of-phase components coupled to the two patches through the two T-shaped probes can produce in-phase radiation at both of the radiating patches. The out-of-phase feeding network, Tx and Rx BSFs, and the $\lambda / 4$ transformed lines constitute the PDDN. The 


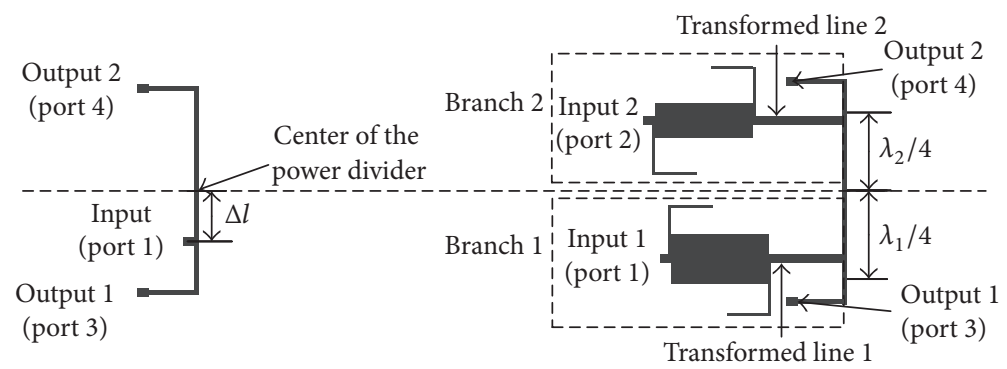

(a)

(b)

FIgURE 5: (a) Layout of the power divider. (b) Layout of the PDDN.

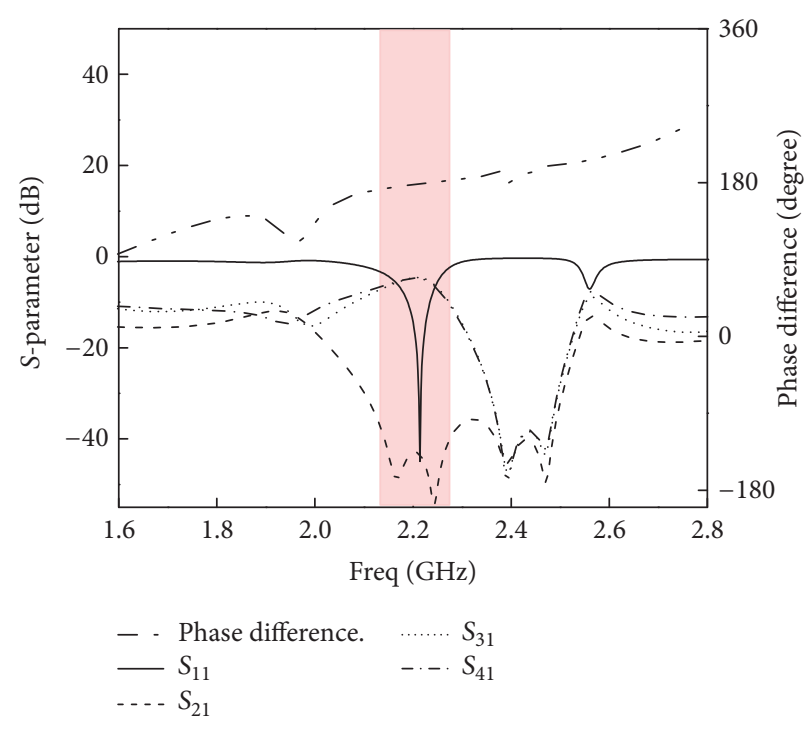

(a)

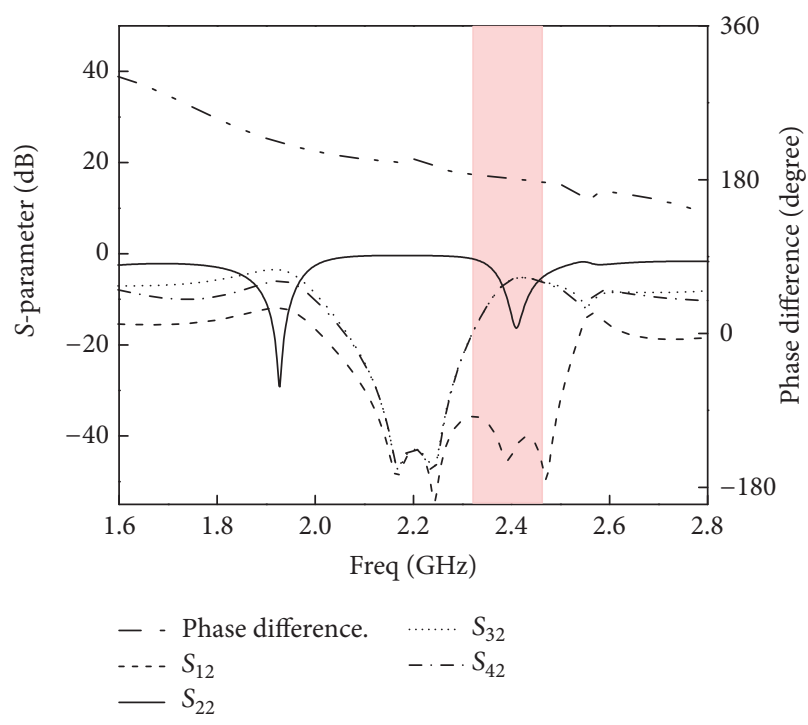

(b)

FIgURE 6: Simulated S-parameters and phase differences between the two output ports of the PDDN: (a) port 1 excited; (b) port 2 excited.

phase difference between port 3 and port 4 of the PDDN is illustrated in Figure 6 . As expected, $180^{\circ}$ phase difference at the operating band of the excited port is obtained between port 3 and port 4 for port 1 or port 2 exited. Moreover, with reference to Figure 6, it can be seen that the PDDN can offer $45 \mathrm{~dB}$ isolation between the two input ports.

2.4. Design of the T-Shaped Coupled Patch Antenna. In this section, two T-shaped probes coupled patches proposed in [9] are designed as the radiating elements of the antenna array. The configuration of the T-shaped probe coupled patch element is shown in Figure 7. The patch element together with the T-shaped probe can be equivalent to a two-order bandpass filter. When the coupling strengths between the Tshaped probes and the patches are proper, the two poles can be achieved. Thus, the operating band can be widened. As a result, the T-shaped probe coupled patch antenna designed as the radiating element is a good choice.

\section{Simulated and Measured Results}

To demonstrate the idea, the integrated filtering microstrip duplex antenna array is designed and fabricated. Figure 8

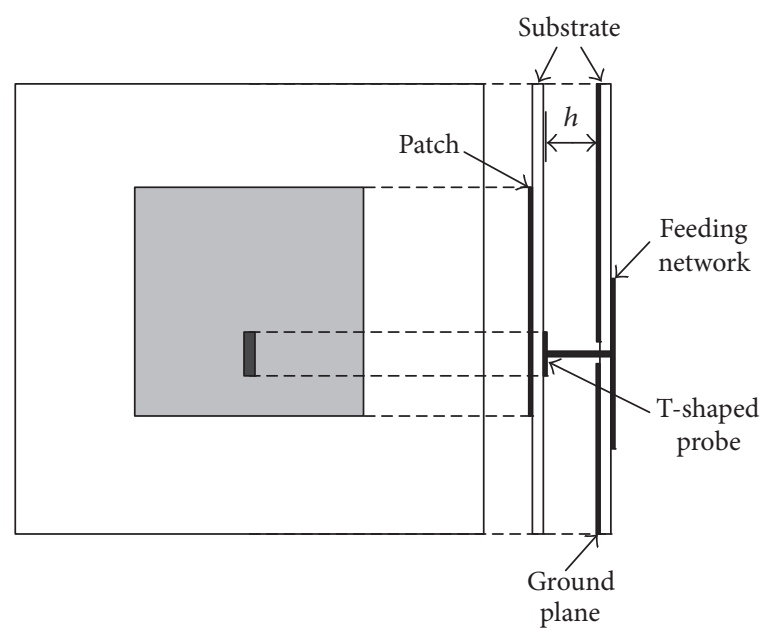

FIGURE 7: Layout of the T-shaped probe coupled antenna.

illustrates the fabricated prototype. Figure 9 shows the simulated and measured reflection and transmission coefficients of the proposed antenna array. It is observed that the measured results agree well with the simulation ones, 

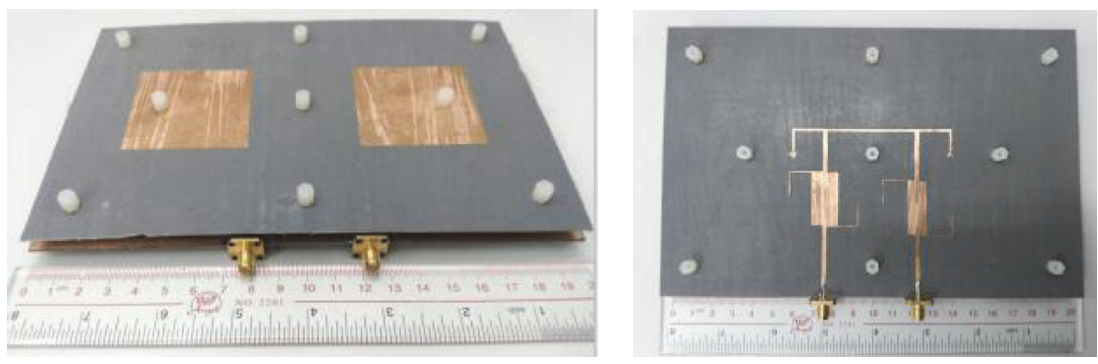

FIGURE 8: Prototype of the fabricated antenna array.

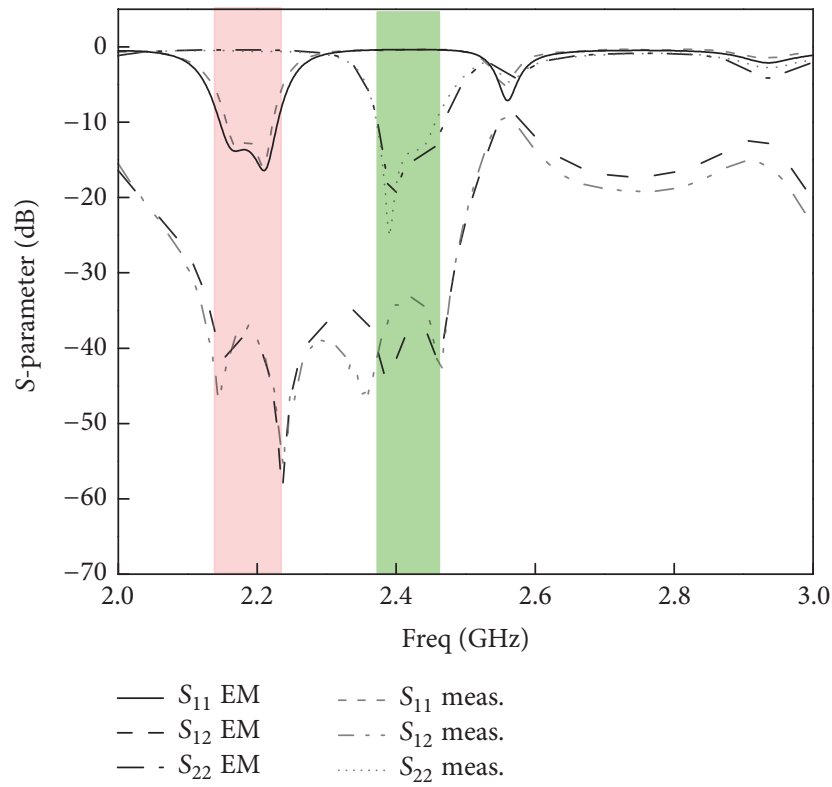

FIGURE 9: Simulated and measured $S$-parameter of the antenna array.

except a small difference caused by the fabrication errors. The fractional bandwidths of the two operating bands are given by $3.1 \%(2.15-2.22 \mathrm{GHz})$ and $3.5 \%(2.37-2.46 \mathrm{GHz})$, respectively. And the port-to-port isolation is better than $35 \mathrm{~dB}$ over the two bands.

The simulated and measured realized gains of the antenna array are shown in Figure 10. It is observed that the antenna array has a flat gain about $9.8 \mathrm{dBi}$ from $2.15 \mathrm{GHz}$ to $2.22 \mathrm{GHz}$ for port 1 excited. This drops sharply to below $-25 \mathrm{dBi}$ at the $2.4 \mathrm{GHz}$ band. When port 2 is excited, the antenna has a stable gain about $10 \mathrm{dBi}$ from $2.37 \mathrm{GHz}$ to $2.46 \mathrm{GHz}$. The gain is suppressed to below $-30 \mathrm{dBi}$ at the $2.2 \mathrm{GHz}$ band.

Figure 11 shows the simulated and measured normalized co- and cross-polarization radiation patterns of the antenna array in two orthogonal planes at $2.2 \mathrm{GHz}$ for port 1 excited and port 2 terminated with a $50 \Omega$ load. The measured and simulated results agree well. The maximum radiation direction can be steered by pattern reconfiguration $[10,11]$. The proposed antenna exhibits a maximum radiation in the broadside direction with the polarization in the $y$-axis. And the measured cross-polarization discrimination (XPD) is over $20 \mathrm{~dB}$ in both $\varphi=90^{\circ}$ and $\varphi=0^{\circ}$ planes.

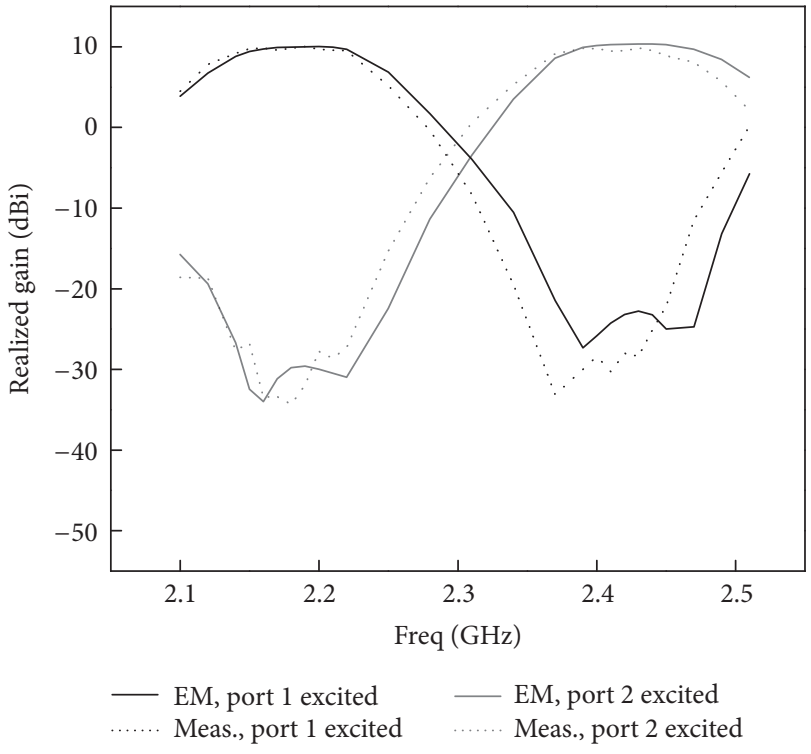

FIGURE 10: Simulated and measured realized antenna gain.

Figure 12 shows the normalized simulated and measured $2.4 \mathrm{GHz}$ radiation patterns for port 2 excited and port 1 terminated with a $50 \Omega$ load. The radiation patterns also exhibit a maximum radiation in the broadside direction with the same polarization in the $y$-axis. The measured XPD is over $28 \mathrm{~dB}$ in $\varphi=90^{\circ}$ and $\varphi=0^{\circ}$ planes.

The simulated antenna efficiencies at $2.2 \mathrm{GHz}$ and $2.4 \mathrm{GHz}$ are about $73 \%$ and $75 \%$, respectively. Table 2 summarized the performances of the proposed antenna compared with other reported antennas in the literature. It can be observed that the proposed antenna array shows good performances of high gain, high isolation, and low crosspolarization level.

\section{Conclusion}

An integrated filtering duplex microstrip antenna array with high isolation has been presented in this paper. The antenna array is composed of two T-shaped probes coupled patch elements and a PDDN. The PDDN is used as the powersplitter and isolated configuration. The lossless TL model is adopted to design the BSFs in PDDN. And the analysis of the PDDN is presented in detail. The simulated and measured 
TABLE 2: Comparison with the previous works.

\begin{tabular}{lcccc}
\hline Antennas & Frequency & Isolation & Gain & XPD \\
\hline$[3]$ & $2.45 / 5.5$ & $>26$ & $1.3 / 4.8$ & $25 / 13$ \\
{$[4]$} & $8.98 / 11.22$ & $>22$ & $4.3 / 4.2$ & $13 / 19$ \\
{$[5]$} & $2.45 / 5.5$ & $>21$ & $2.3 / 3.6$ & $21.8 / 18.3$ \\
{$[6]$} & $2.58 / 2.87$ & $>32$ & $5 / 4.5$ & $20 / 28$ \\
This work & $2.2 / 2.4$ & $>35$ & $9.8 / 10$ & $20 / 28$ \\
\hline
\end{tabular}
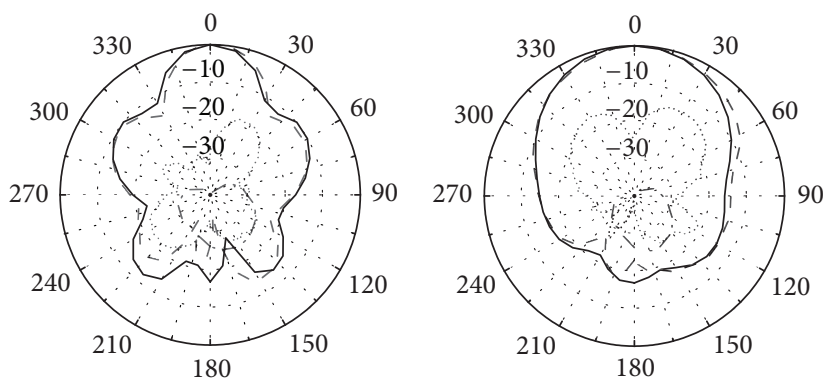

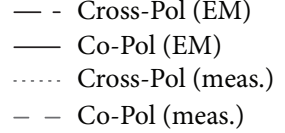

(a)

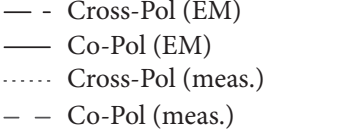

(b)

FIGURE 11: Normalized radiation patterns at $2.2 \mathrm{GHz}$ for port 1 excited: (a) $\varphi=90^{\circ}$; (b) $\varphi=0^{\circ}$.

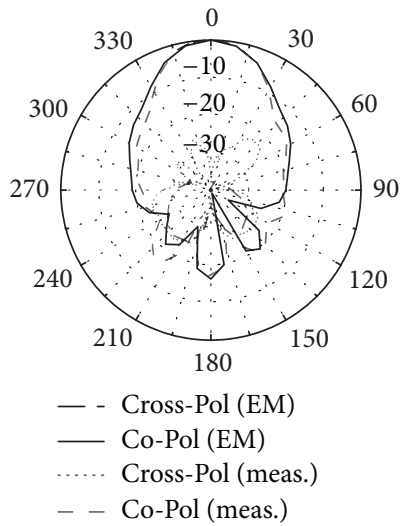

(a)
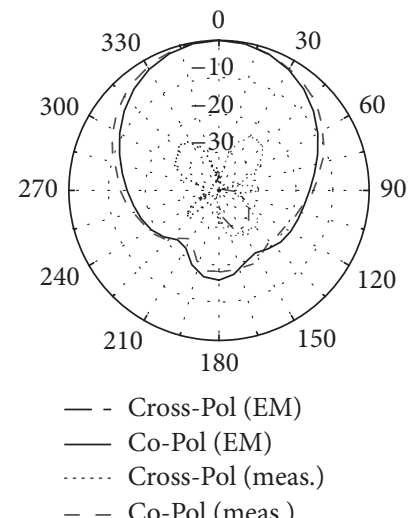

(b)

FIGURE 12: Normalized radiation patterns at $2.4 \mathrm{GHz}$ for port 2 excited: (a) $\varphi=90^{\circ}$; (b) $\varphi=0^{\circ}$.

results agree well, showing good performances of impedance matching, gain, ports isolation, XPD, and similar radiation pattern.

\section{Competing Interests}

The authors declare that there is no conflict of interests regarding the publication of this paper.

\section{Acknowledgments}

This work is supported by the National Natural Science Foundation of China (61571194).

\section{References}

[1] M. Nakano, H. Arai, W. Chujo, M. Fujise, and N. Goto, "Feed circuits of double-layered self-diplexing antenna for mobile satellite communications," IEEE Transactions on Antennas and Propagation, vol. 40, no. 10, pp. 1269-1271, 1992.

[2] C.-X. Mao, S. Gao, Y. Wang, F. Qin, and Q.-X. Chu, "Multimode resonator-fed dual-polarized antenna array with enhanced bandwidth and selectivity," IEEE Transactions on Antennas and Propagation, vol. 63, no. 12, pp. 5492-5499, 2015.

[3] Y. J. Lee, J. H. Tarng, and S. J. Chung, "A filtering diplexing antenna for dual-band operation with similar radiation patterns and low cross-polarization levels," IEEE Antennas and Wireless Propagation Letters, In press. 
[4] Y. Zhang, X. Y. Zhang, L.-H. Ye, and Y.-M. Pan, "Dual-band base station array using filtering antenna elements for mutual coupling suppression," IEEE Transactions on Antennas and Propagation, vol. 64, no. 8, pp. 3423-3430, 2016.

[5] C.-X. Mao, S. Gao, Y. Wang, F. Qin, and Q.-X. Chu, "Compact highly integrated planar duplex antenna for wireless communications," IEEE Transactions on Microwave Theory and Techniques, vol. 64, no. 7, pp. 2006-2013, 2016.

[6] F.-C. Chen, N.-Y. Zhang, P.-S. Zhang, and Q.-X. Chu, "Design of ultra-wideband bandstop filter using defected ground structure," Electronics Letters, vol. 49, no. 16, pp. 1010-1011, 2013.

[7] Y.-C. Lu and Y.-C. Lin, "A mode-based design method for dualband and self-diplexing antennas using double T-stubs loaded aperture," IEEE Transactions on Antennas and Propagation, vol. 60, no. 12, pp. 5596-5603, 2012.

[8] S. Mukherjee and A. Biswas, "Design of self-diplexing substrate integrated waveguide cavity backed slot antenna," IEEE Antennas and Wireless Propagation Letters, vol. 15, pp. 1775-1778, 2016.

[9] C. L. Mak, K. F. Lee, and K. M. Luk, "Broadband patch antenna with a T-shaped probe," IEE Proceedings-Microwaves, Antennas and Propagation, vol. 147, no. 2, pp. 73-76, 2000.

[10] L. Gong, K. Y. Chan, and R. Ramer, "A beam steering singlearm rectangular spiral antenna with large azimuth space coverage," in Proceedings of the IEEE 14th Annual Wireless and Microwave Technology Conference (WAMICON '13), pp. 1-4, IEEE, Orlando, Fla, USA, April 2013.

[11] L. Gong, Y. Yang, K. Y. Chan, and R. Ramer, "RHCP patternreconfigurable spiral antenna biased with two DC signals," Microwave and Optical Technology Letters, vol. 56, no. 7, pp. 1636-1640, 2014. 


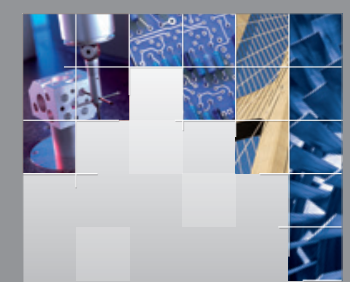

\section{Enfincering}
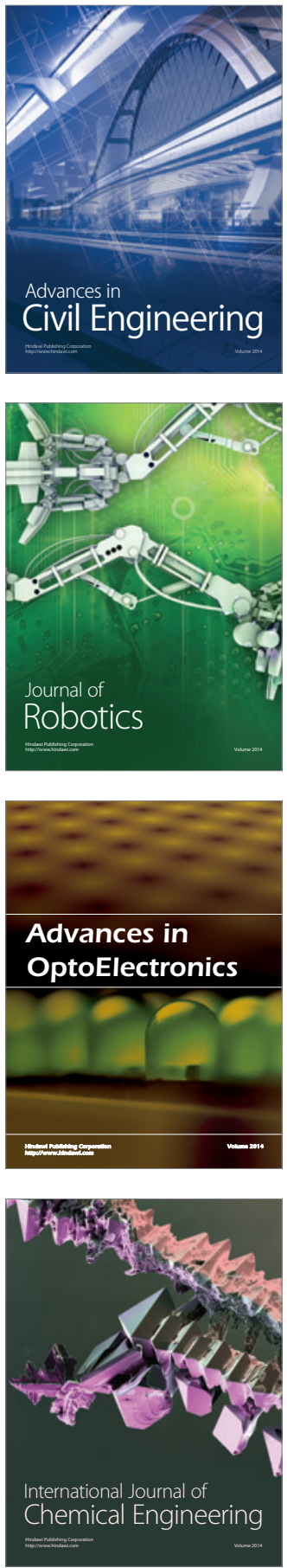

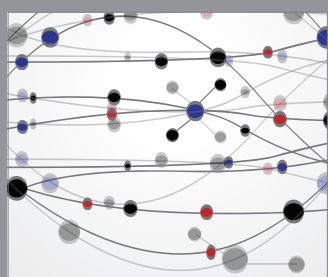

The Scientific World Journal

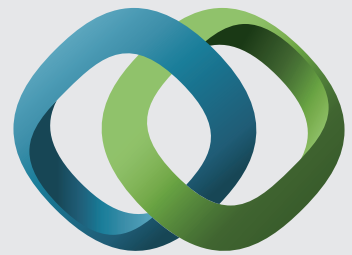

\section{Hindawi}

Submit your manuscripts at

https://www.hindawi.com
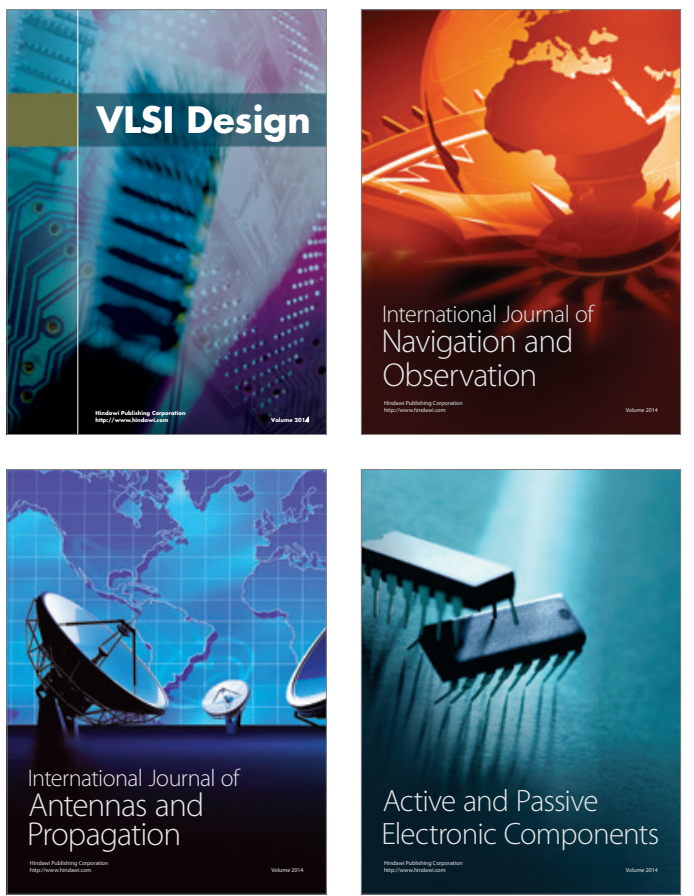
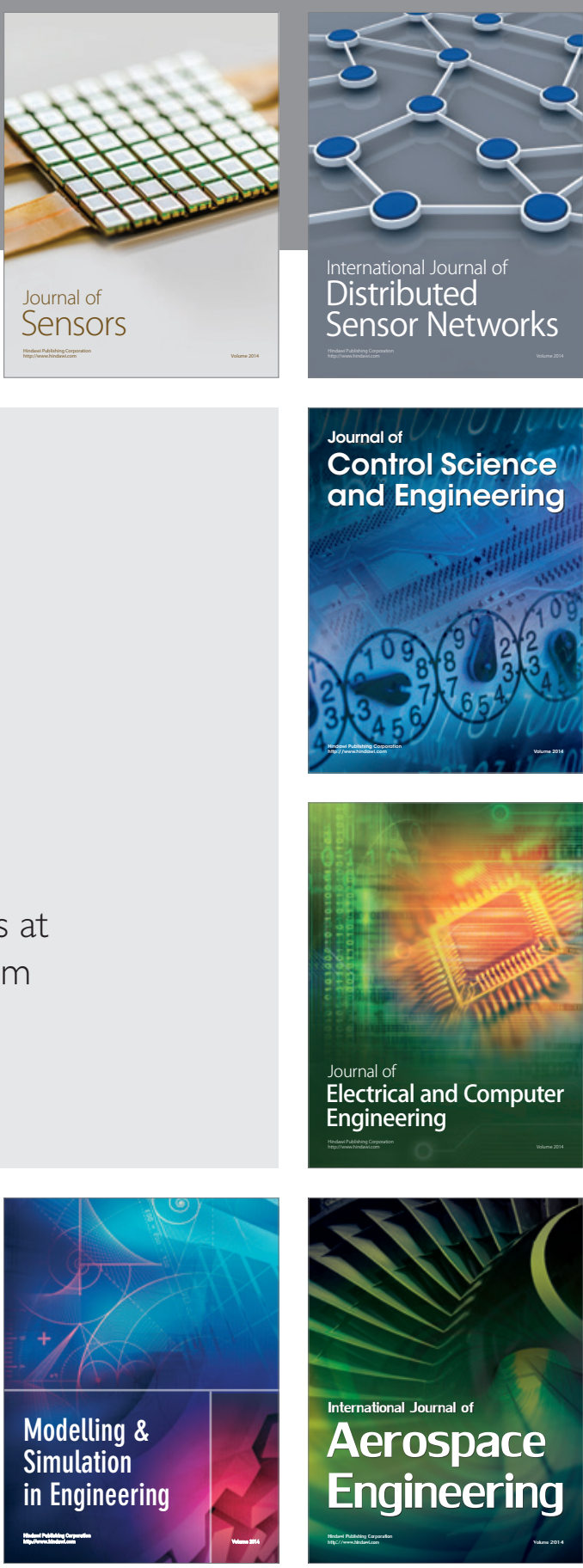

International Journal of

Distributed

Sensor Networks

$-$

Joumal of

Control Science

and Engineering
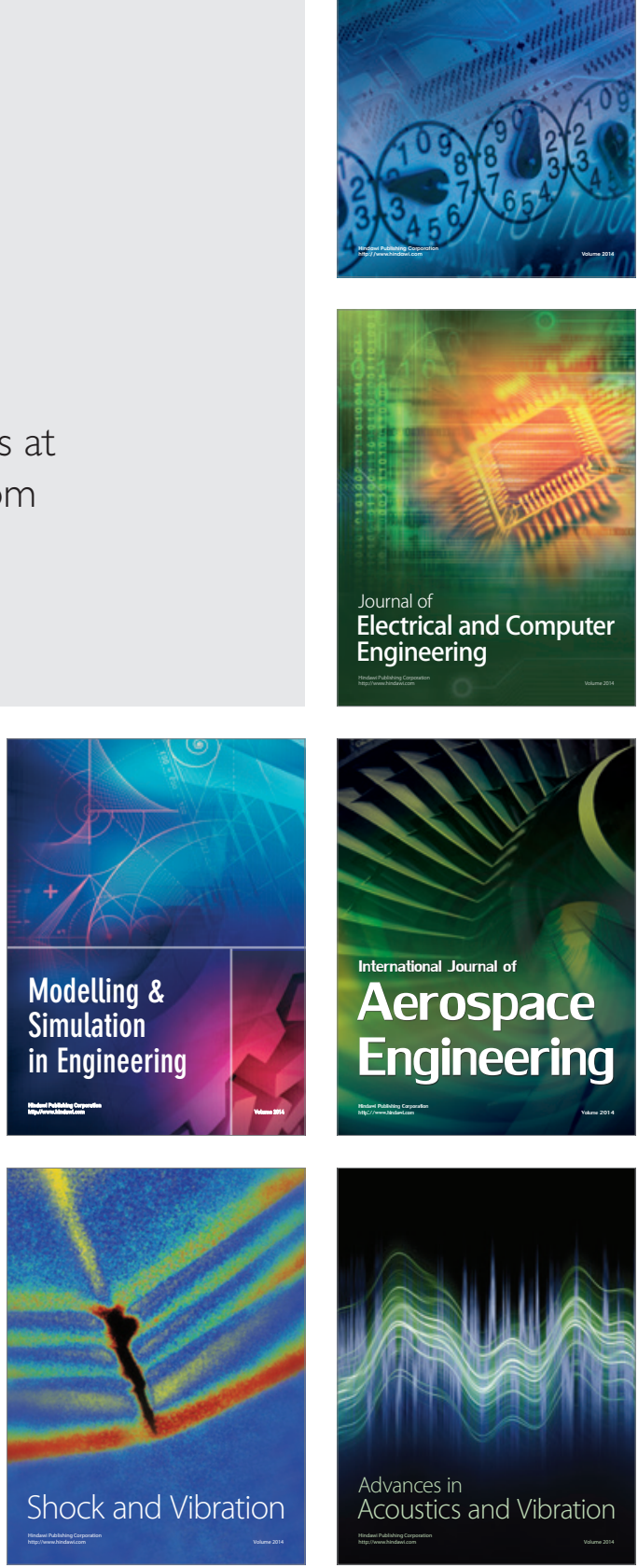